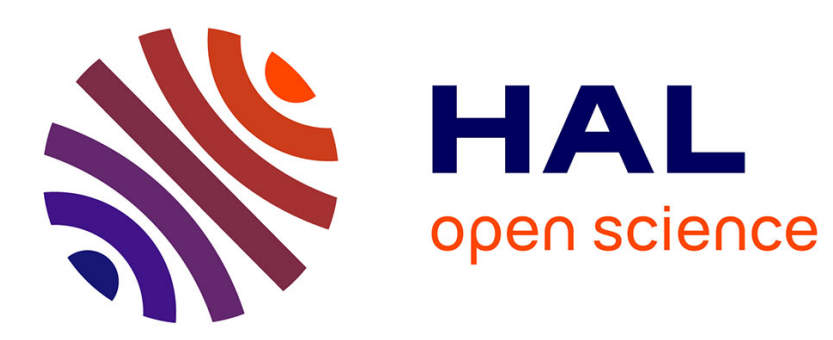

\title{
Profile of blowing-up solutions to a nonlinear system of fractional differential equations
}

\author{
Mokhtar Kirane, Salman Amin Malik
}

\section{To cite this version:}

Mokhtar Kirane, Salman Amin Malik. Profile of blowing-up solutions to a nonlinear system of fractional differential equations. 2010. hal-00467202

\author{
HAL Id: hal-00467202 \\ https://hal.science/hal-00467202 \\ Preprint submitted on 26 Mar 2010
}

HAL is a multi-disciplinary open access archive for the deposit and dissemination of scientific research documents, whether they are published or not. The documents may come from teaching and research institutions in France or abroad, or from public or private research centers.
L'archive ouverte pluridisciplinaire HAL, est destinée au dépôt et à la diffusion de documents scientifiques de niveau recherche, publiés ou non, émanant des établissements d'enseignement et de recherche français ou étrangers, des laboratoires publics ou privés. 


\title{
Profile of blowing-up solutions to a nonlinear system of fractional differential equations
}

\author{
Mokhtar Kirane, Salman A. Malik \\ Laboratoire de Mathématiques, Image et Applications, Université de La Rochelle, Avenue M. \\ Crépeau, 17042 La Rochelle Cedex, France.
}

Abstract

We investigate the profile of the blowing up solutions to the nonlinear nonlocal system (FDS)

$$
\begin{aligned}
u^{\prime}(t)+D_{0_{+}}^{\alpha}\left(u-u_{0}\right)(t) & =|v(t)|^{q}, & t>0, \\
v^{\prime}(t)+D_{0_{+}}^{\beta}\left(v-v_{0}\right)(t) & =|u(t)|^{p}, & t>0,
\end{aligned}
$$

where $u(0)=u_{0}>0, v(0)=v_{0}>0, p>1, q>1$ are given constants and $D_{0_{+}}^{\alpha}$ and $D_{0_{+}}^{\beta}, 0<\alpha<1,0<\beta<1$ stand for the Riemann-Liouville fractional derivatives. Our method of proof relies on comparisons of the solution to the (FDS) with solutions of the subsystems obtained from (FDS) by dropping either the usual derivatives or the fractional derivatives.

Key words: Nonlinear system, Fractional derivative, Integral equations, Blow up time, Laplace transform.

2010 MSC: 26A33, 45J05, 34K37.

\section{Introduction}

In this paper, we are concerned with the profile of the blowing up solutions to the nonlinear system of fractional differential equations (FDS)

$$
\begin{aligned}
u^{\prime}(t)+D_{0_{+}}^{\alpha}\left(u-u_{0}\right)(t) & =|v(t)|^{q}, & t>0, \\
v^{\prime}(t)+D_{0_{+}}^{\beta}\left(v-v_{0}\right)(t) & =|u(t)|^{p}, & t>0,
\end{aligned}
$$

Email addresses: mokhtar.kirane@univ-lr.fr (Mokhtar Kirane), salman.malik@univ-lr.fr (Salman A. Malik)

Preprint submitted to nonlinear analysis 
subject to the initial conditions

$$
u(0)=u_{0}, \quad v(0)=v_{0},
$$

where $p>1, q>1, u(0)=u_{0}>0, v(0)=v_{0}>0$ are constants, $D_{0_{+}}^{\alpha}$ and $D_{0_{+}}^{\beta}$ stand for the Riemann-Liouville fractional derivatives of orders $0<\alpha<1$ and $0<\beta<1$, respectively.

It has been proven by Furati and Kirane in [1] that, under the constraint 1 $1 / p q \leq \alpha+\beta / p$ or $1-1 / p q \leq \beta+\alpha / q$, any solution emerging from $\left(u_{0}, v_{0}\right),\left(u_{0}>\right.$ $\left.0, v_{0}>0\right)$ blows up in a finite time. Moreover, they gave an upper bound for the blow up time. Their method of proof relies on the weak formulation of the system (FDS) with a judicious choice of the test function. Here, we present estimates from above and below of the blowing up solutions by comparing them with the solutions of the particular systems obtained from (FDS):

$$
\left\{\begin{array}{lll}
u^{\prime}(t)=\lambda|v(t)|^{q}, & t>0, & p>1, \\
v^{\prime}(t)=\lambda|u(t)|^{p}, & t>0, & q>1,
\end{array}\right.
$$

the system of ordinary differential equations (ODS) with either $\lambda=1$ or $\lambda=1 / 2$, and

$$
\begin{cases}D_{0_{+}}^{\alpha}\left(u-u_{0}\right)(t)=\mu|v(t)|^{q}, & t>0 \\ D_{0_{+}}^{\beta^{\prime}}\left(v-v_{0}\right)(t)=\mu|u(t)|^{p}, & t>0\end{cases}
$$

the system of fractional differential equations (PFDS), with either $\mu=1$ or $\mu=$ $1 / 2$. As the solutions to (ODS) are easy to obtain, we will investigate in particular the system (PFDS).

Our paper is organized as follows: some definitions and known results needed in the sequel are stated in the next section. Section 3 is devoted to our main results for the investigation of the profile of the blowing up solutions of system (FDS). In the last section, we provide numerical approximations of the solutions, and the profiles are given for particular examples.

\section{Preliminaries}

In this section, the necessary definitions and notations from fractional calculus needed for the paper are recalled for the sake of the reader.

The Riemann-Liouville fractional integral of order $0<\alpha<1$ is

$$
J_{0_{+}}^{\alpha} f(t):=\frac{1}{\Gamma(\alpha)} \int_{0}^{t} \frac{f(\tau)}{(t-\tau)^{1-\alpha}} d \tau, \quad t>0,
$$


where $\Gamma(\alpha)$ is the Euler Gamma function and $f: \mathbb{R}^{+} \rightarrow \mathbb{R}$ is a real valued integrable function. The integral (4) can be written as a convolution

$$
J_{0_{+}}^{\alpha} f(t)=\left(\phi_{\alpha} \star f\right)(t)
$$

where

$$
\phi_{\alpha}:= \begin{cases}\frac{t^{\alpha-1}}{\Gamma(\alpha)}, & t \geq 0 \\ 0 & t<0 .\end{cases}
$$

The Laplace transform of the Riemann-Liouville integral of order $0<\alpha<1$ is

$$
\mathcal{L}\left\{J_{0_{+}}^{\alpha} f(t): s\right\}=\mathcal{L}\{f(t): s\} / s^{\alpha} .
$$

The Riemann-Liouville fractional derivative of order $0<\alpha<1$ is

$$
D_{0_{+}}^{\alpha} f(t):=\frac{d}{d t} J_{0_{+}}^{1-\alpha} f(t)=\frac{1}{\Gamma(1-\alpha)} \frac{d}{d t} \int_{0}^{t} \frac{f(\tau)}{(t-\tau)^{\alpha}} d \tau
$$

In particular, when $\alpha=0, D_{0_{+}}^{0} f(t)=f(t)$; note that the Riemann-Liouville fractional derivative of a constant is not equal to zero.

The Caputo fractional derivative of order $0<\alpha<1$ is defined by

$$
{ }^{C} D_{0_{+}}^{\alpha} f(t):=J_{0_{+}}^{1-\alpha} \frac{d}{d t} f(t)=\frac{1}{\Gamma(1-\alpha)} \int_{0}^{t} \frac{f^{\prime}(\tau)}{(t-\tau)^{\alpha}} d \tau .
$$

The relation between the Riemann-Liouville and the Caputo fractional derivatives is

$$
{ }^{C} D_{0_{+}}^{\alpha} f(t)=D_{0_{+}}^{\alpha}(f(t)-f(0)), \quad 0<\alpha<1 .
$$

The formula for the integration by parts in $[0, T]$ is given by

$$
\int_{0}^{T} f(t) D_{0_{+}}^{\alpha} g(t) d t=\int_{0}^{T} D_{T_{-}}^{\alpha} f(t) g(t) d t
$$

see [2], where $D_{T_{-}}^{\alpha}$ is the right sided fractional derivative defined by

$$
D_{T_{-}}^{\alpha} f(t):=-\frac{1}{\Gamma(1-\alpha)} \frac{d}{d t} \int_{t}^{T} \frac{f(\tau)}{(\tau-t)^{\alpha}} d \tau, \quad 0<\alpha<1 .
$$


The Laplace transforms of the Riemann-Liouville derivative $D_{0_{+}}^{\alpha} f(t)$ and the Caputo derivative ${ }^{C} D_{0_{+}}^{\alpha} f(t)$ are

$$
\begin{gathered}
\mathcal{L}\left\{D_{0_{+}}^{\alpha} f(t): s\right\}=s^{\alpha} \mathcal{L}\{f(t): s\}-J_{0_{+}}^{1-\alpha} f(0), \quad 0<\alpha<1, \\
\mathcal{L}\left\{{ }^{C} D_{0_{+}}^{\alpha} f(t): s\right\}=s^{\alpha} \mathcal{L}\{f(t): s\}-s^{\alpha-1} f(0), \quad 0<\alpha<1 .
\end{gathered}
$$

At this stage let us recall the test function considered in [1]:

$$
\varphi(t)= \begin{cases}T^{-\lambda}(T-t)^{\lambda}, & t \in[0, T] \\ 0 & t>T\end{cases}
$$

it satisfies

$$
\int_{0}^{T} D_{T_{-}}^{\alpha} \varphi(t) d t=C_{\alpha, \lambda} T^{1-\alpha}, \quad C_{\alpha, \lambda}=\frac{\Gamma(\lambda+1)}{\Gamma(\lambda-\alpha+2)},
$$

and for $p>1, \lambda>\alpha p-1$

$$
\int_{0}^{T} \varphi^{1-p}(t)\left|D_{T-}^{\alpha} \varphi(t)\right|^{p} d t=C_{p, \alpha} T^{1-\alpha p}
$$

where

$$
C_{p, \alpha}=\frac{1}{\lambda-p \alpha+1}\left[\frac{\Gamma(\lambda+1)}{\Gamma(\lambda+1-\alpha)}\right]^{p} .
$$

The main result in [1] is the

Theorem 2.1 ([1]). Suppose that $0<\alpha, \beta<1, p, q>1$ and $u_{0}>0, v_{0}>0$, then solutions to system (FDS) subject to (1) blow up in a finite time, whenever

$$
1-\frac{1}{p q} \leq \alpha+\frac{\beta}{p}, \quad \text { or } \quad 1-\frac{1}{p q} \leq \beta+\frac{\alpha}{q} .
$$

Let us recall that the blowing up solution of the system (ODS) with $\lambda=1$

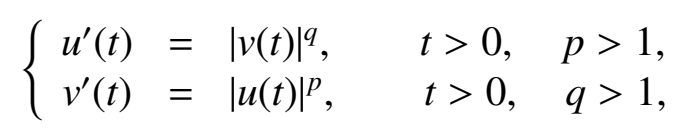

subject to the initial conditions $\left(u(0)=u_{0}>0, v(0)=v_{0}>0\right)$ is

$$
u_{o d}(t)=C_{1}\left(T_{\max }-t\right)^{-\frac{q+1}{p q-1}}, \quad v_{o d}(t)=C_{2}\left(T_{\max }-t\right)^{-\frac{p+1}{p q-1}},
$$


where $T_{\max }, C_{1}$ and $C_{2}$ are given by

$$
\begin{aligned}
T_{\text {max }} & =\left(\frac{C_{2} u_{0}}{C_{1} v_{0}}\right)^{\frac{p q-1}{p-q}}, \\
C_{1} & =\left(\frac{(p+1)^{q}(q+1)}{(p q-1)^{q+1}}\right)^{\frac{1}{p q-1}}, \quad C_{2}=\left(\frac{(p+1)(q+1)^{p}}{(p q-1)^{p+1}}\right)^{\frac{1}{p q-1}} .
\end{aligned}
$$

Alike, the blowing up solution of the system (ODS) with $\lambda=1 / 2$

$$
\left\{\begin{array}{lll}
u^{\prime}(t)=\frac{1}{2}|v(t)|^{q}, & t>0, & u(0)=u_{0}>0 \\
v^{\prime}(t)=\frac{1}{2}|u(t)|^{p}, & t>0, & v(0)=v_{0}>0
\end{array}\right.
$$

for $p, q>1$, is given by

$$
u_{o d}^{*}(t)=C_{1}^{*}\left(T_{\text {max }}^{*}-t\right)^{-\frac{q+1}{p q-1}}, \quad v_{o d}^{*}(t)=C_{2}^{*}\left(T_{\max }^{*}-t\right)^{-\frac{p+1}{p q-1}},
$$

where $T_{\max }^{*}, C_{1}^{*}$ and $C_{2}^{*}$ are given by

$$
\begin{aligned}
T_{\text {max }}^{*} & =\left(\frac{C_{2}^{*} u_{0}}{C_{1}^{*} v_{0}}\right)^{\frac{p q-1}{p-q}}, \\
C_{1}^{*} & =2^{\frac{q+1}{p q-1}}\left(\frac{(p+1)^{q}(q+1)}{(p q-1)^{q+1}}\right)^{\frac{1}{p q-1}}, \quad C_{2}^{*}=2^{\frac{p+1}{p q-1}}\left(\frac{(p+1)(q+1)^{p}}{(p q-1)^{p+1}}\right)^{\frac{1}{p q-1}} .
\end{aligned}
$$

Notice that the solution of the system (18) blows up earlier than the solution of the system (21) since $T_{\text {max }}^{*}=2 T_{\max }$.

\section{Main Results}

In this section, we consider the nonlinear system of fractional differential equations (PFDS)

$$
\begin{array}{ll}
D_{0_{+}}^{\alpha}\left(u-u_{0}\right)(t)=\mu|v(t)|^{q}, & t>0, \\
D_{0_{+}}^{\beta}\left(v-v_{0}\right)(t)=\mu|u(t)|^{p}, & t>0,
\end{array}
$$

for $0<\alpha, \beta<1$, with either $\mu=1$ or $\mu=1 / 2$, subject to the initial conditions (1). For $\mu=1$ we will give the necessary conditions for solutions to blow up in a finite time; moreover an upper bound on the blow up time is provided.

Let us set

$$
s=\frac{p \alpha+\beta}{1-p q}, \quad \tilde{s}=\frac{\alpha+q \beta}{1-p q}, \quad C_{v}=K_{2}\left(K_{1}\right)^{\frac{p}{p q-1}}, \quad C_{u}=K_{2}^{*}\left(K_{1}^{*}\right)^{\frac{q}{p q-1}},
$$


where $K_{1}, K_{2}, K_{1}^{*}$ and $K_{2}^{*}$ are:

$$
\begin{aligned}
& K_{1}=C_{p^{\prime}, \alpha}^{\frac{1}{p^{\prime}}} C_{q^{\prime}, \beta}^{\frac{1}{p q^{\prime}}}, \quad K_{2}=C_{q^{\prime}, \beta}^{\frac{1}{q^{\prime}}} C_{\beta, \lambda}^{-1}, \\
& K_{1}^{*}=C_{q^{\prime}, \beta}^{\frac{1}{q^{\prime}}} C_{p^{\prime}, \alpha}^{\frac{1}{q p^{\prime}}}, \quad K_{2}^{*}=C_{p^{\prime}, \alpha}^{\frac{1}{p^{\prime}}} C_{\alpha, \lambda}^{-1},
\end{aligned}
$$

and the constants $\left\{C_{\alpha, \lambda}, C_{\beta, \lambda}\right\}$ and $\left\{C_{p^{\prime}, \alpha}, C_{q^{\prime}, \beta}\right\}$ are given by (15) and (17) respectively.

The first result is given by

Theorem 3.1. Let $p, q>1$ and $u_{0}>0, v_{0}>0$. If

$$
1-\frac{1}{p q} \leq \alpha+\frac{\beta}{p}, \quad \text { or } \quad 1-\frac{1}{p q} \leq \beta+\frac{\alpha}{q},
$$

then any solution to the system (PFDS) with $\mu=1$ subject to the initial conditions (1) blows up in a finite time $T_{\max }$. Furthermore, an upper bound on the blow up time $T_{\text {max }}$ is given by $\min \left\{T_{u}, T_{v}\right\}$ where

$$
T_{v}=\left[\frac{v_{0}}{C_{v}}\right]^{1 / s} \quad T_{u}=\left[\frac{u_{0}}{C_{u}}\right]^{1 / \tilde{s}}
$$

Proof. The proof is by contradiction; suppose $(u, v)$ is a global solution of the system (PFDS). Multiplying the equations of system (PFDS) by $\varphi$ and integrating over $[0, T]$, we, by virtue of $(10)$, obtain

$$
\begin{aligned}
& \int_{0}^{T} u D_{T_{-}}^{\alpha} \varphi=u_{0} \int_{0}^{T} D_{T_{-}}^{\alpha} \varphi+\int_{0}^{T}|v|^{q} \varphi, \\
& \int_{0}^{T} v D_{T_{-}}^{\beta} \varphi=v_{0} \int_{0}^{T} D_{T_{-}}^{\beta} \varphi+\int_{0}^{T}|u|^{p} \varphi,
\end{aligned}
$$

for any function $\varphi$ such that $D_{T_{-}}^{\alpha} \varphi$ exists and $\varphi(T)=0$. Using Hölder's inequality, we obtain

$$
\begin{aligned}
& \int_{0}^{T} u D_{T_{-}}^{\alpha} \varphi \leq\left[\int_{0}^{T}|u|^{p} \varphi\right]^{1 / p}\left[\int_{0}^{T}\left|D_{T_{-}}^{\alpha} \varphi\right|^{p^{\prime}} \varphi^{-p^{\prime} / p}\right]^{1 / p^{\prime}}, \\
& \int_{0}^{T} v D_{T_{-}}^{\beta} \varphi \leq\left[\int_{0}^{T}|v|^{q} \varphi\right]^{1 / q}\left[\int_{0}^{T}\left|D_{T_{-}}^{\beta} \varphi\right|^{q^{\prime}} \varphi^{-q^{\prime} / q}\right]^{1 / q^{\prime}},
\end{aligned}
$$


where $p, p^{\prime}, q, q^{\prime}$ satisfy $p+p^{\prime}=p p^{\prime}$ and $q+q^{\prime}=q q^{\prime}$. If we set

$$
\begin{aligned}
\mathbf{I}:=\int_{0}^{T}|u|^{p} \varphi, & \mathbf{J}:=\int_{0}^{T}|v|^{q} \varphi, \\
\mathbf{A}:=\int_{0}^{T}\left|D_{T_{-}}^{\alpha} \varphi\right|^{p^{\prime}} \varphi^{-p^{\prime} / p}, & \mathbf{B}:=\int_{0}^{T}\left|D_{T_{-}}^{\beta} \varphi\right|^{q^{\prime}} \varphi^{-q^{\prime} / q},
\end{aligned}
$$

then (22), (23), (24) and (25) lead to:

$$
\mathbf{J} \leq \mathbf{I}^{1 / p} \mathbf{A}^{1 / p^{\prime}} \text { and } \quad \mathbf{I} \leq \mathbf{J}^{1 / q} \mathbf{B}^{1 / q^{\prime}} .
$$

As $u_{0}>0, v_{0}>0$ and $\int_{0}^{T} D_{T_{-}}^{\alpha} \varphi>0$ when $\varphi(t)$ is selected as in (14), it is clear that

$$
\begin{aligned}
& \mathbf{J}^{1-\frac{1}{p q}} \leq \mathbf{A}^{1 / p^{\prime}} \mathbf{B}^{1 / p q^{\prime}}, \\
& \mathbf{I}^{1-\frac{1}{p q}} \leq \mathbf{A}^{1 / q p^{\prime}} \mathbf{B}^{1 / q^{\prime}} .
\end{aligned}
$$

Using (16), with $\lambda \geq \max \left\{\alpha p^{\prime}-1, \beta q^{\prime}-1\right\}$, we have

$$
\mathbf{A}=C_{p^{\prime}, \alpha} T^{1-\alpha p^{\prime}}, \quad \mathbf{B}=C_{q^{\prime}, \beta} T^{1-\beta q^{\prime}} .
$$

Consequently, (27) takes the form

$$
\mathbf{J}^{1-\frac{1}{p q}} \leq K_{1} T^{s_{1}}
$$

where

$$
s_{1}=1-\alpha-\frac{\beta}{p}-\frac{1}{p q} .
$$

Thus $\mathbf{J}$ is bounded under the constraint $s_{1} \leq 0$ which is equivalent to

$$
1-\frac{1}{p q} \leq \alpha+\frac{\beta}{p}
$$

The equality (23) and the estimates (25) allow us to write

$$
v_{0} \int_{0}^{T} D_{T_{-}}^{\beta} \varphi \leq \mathbf{J}^{1 / q} \mathbf{B}^{1 / q^{\prime}},
$$

which in virtue of (30) becomes

$$
v_{0} \leq \underset{7}{K_{2} \mathbf{J}^{1 / q}} T^{r_{1}}
$$


where $r_{1}=-1 / q$.

Letting $T \rightarrow \infty$, in (31) we obtain the contradiction $0<v_{0} \leq 0$. Similarly, an analysis could be done along $\mathbf{I}$; it leads to

$$
1-\frac{1}{p q} \leq \beta+\frac{\alpha}{q}
$$

To obtain an estimation on the blow up time, we estimate $\mathbf{J}$ in (31) by (29) to obtain

$$
\begin{aligned}
v_{0} & \leq K_{2}\left(K_{1}\right)^{\frac{p}{p q-1}} T^{\frac{p s_{1}}{p q-1}+r_{1}} \\
& =C_{v} T^{s}
\end{aligned}
$$

where $s<0$.

Whereupon a bound on the blow-up time is given by

$$
T_{\max } \leq T_{v}=\left[\frac{v_{0}}{C_{v}}\right]^{1 / s} .
$$

A similar bound on $T_{\max }$ can be obtained in terms of $u_{0}$ if we use (22) and (28); it reads

$$
T_{\max } \leq T_{u}=\left[\frac{u_{0}}{C_{u}}\right]^{1 / \tilde{s}} .
$$

Finally, $T_{\max } \leq \operatorname{Min}\left\{T_{u}, T_{v}\right\}$.

Now, we present estimates from below and above of the blowing up solutions to the system (FDS) via the solutions of systems (PFDS) and (ODS) for $\mu=$ $1,1 / 2$ and $\lambda=1,1 / 2$. First observe that system (PFDS) is equivalent to the nonlinear system of Volterra equations

$$
\begin{cases}u(t)=u_{0}+\frac{\mu}{\Gamma(\alpha)} \int_{0}^{t} \frac{|v(\tau)|^{q}}{(t-\tau)^{1-\alpha}} d \tau, & t>0 \\ v(t)=v_{0}+\frac{\mu}{\Gamma(\beta)} \int_{0}^{t} \frac{|u(\tau)|^{p}}{(t-\tau)^{1-\beta}} d \tau, & t>0\end{cases}
$$

Due to the positivity of initial conditions (1), the solution of the system (32) is always greater or equal than the solution of the system

$$
\left\{\begin{array}{cc}
\tilde{u}(t)=\frac{\mu}{\Gamma(\alpha)} \int_{0}^{t} \frac{|\tilde{v}(\tau)|^{q}}{(t-\tau)^{1-\alpha}} d \tau, & t>0 \\
\tilde{v}(t)=\frac{\mu}{\Gamma(\beta)} \int_{0}^{t} \frac{|\tilde{u}(\tau)|^{p}}{(t-\tau)^{1-\beta}} d \tau, & t>0 \\
8 &
\end{array}\right.
$$


with $\mu=1$ or $\mu=1 / 2$. Following [3], growth rates near the blow-up time for blowing up solutions to the system (33) are available. They are stated in

Theorem 3.2. The profiles of the components of the solution $(\tilde{u}(t), \tilde{v}(t))$ for the system (33) with $\mu=1$ are given by

$$
\tilde{u}(t) \sim C_{1, \alpha, \beta}\left(T_{\max }^{\alpha, \beta}-t\right)^{-\delta}, \quad \tilde{v}(t) \sim C_{2, \alpha, \beta}\left(T_{\max }^{\alpha, \beta}-t\right)^{-\xi}, \quad \text { as } \quad t \rightarrow T_{\max }^{\alpha, \beta},
$$

where $T_{\text {max }}^{\alpha, \beta}$ is the blow-up time and

$C_{1, \alpha, \beta}=\left(\frac{\Gamma(p \delta)}{\Gamma(p \delta-\beta)}\right)^{\frac{q}{p q-1}}\left(\frac{\Gamma(q \xi)}{\Gamma(q \xi-\alpha)}\right)^{\frac{1}{p q-1}}, \quad C_{2, \alpha, \beta}=\left(\frac{\Gamma(q \xi)}{\Gamma(q \xi-\alpha)}\right)^{\frac{p}{p q-1}}\left(\frac{\Gamma(p \delta)}{\Gamma(p \delta-\beta)}\right)^{\frac{1}{p q-1}}$,

with

$$
\delta=\frac{\alpha+q \beta}{p q-1}, \quad \xi=\frac{\beta+p \alpha}{p q-1} .
$$

Alike, the profiles of $u(t), v(t)$ for the system (33) with $\mu=1 / 2$ are given by

$$
u(t) \sim C_{1, \alpha, \beta}^{*}\left(\tau_{\max }^{\alpha, \beta}-t\right)^{-\delta}, \quad v(t) \sim C_{2, \alpha, \beta}^{*}\left(\tau_{\max }^{\alpha, \beta}-t\right)^{-\xi}, \quad \text { as } \quad t \rightarrow \tau_{\max }^{\alpha, \beta},
$$

where

$C_{1, \alpha, \beta}^{*}=\left(\frac{\Gamma(p \delta)}{2 \Gamma(p \delta-\beta)}\right)^{\frac{q}{p q-1}}\left(\frac{\Gamma(q \xi)}{\Gamma(q \xi-\alpha)}\right)^{\frac{1}{p q-1}}, \quad C_{2, \alpha, \beta}^{*}=\left(\frac{\Gamma(q \xi)}{2 \Gamma(q \xi-\alpha)}\right)^{\frac{p}{p q-1}}\left(\frac{\Gamma(p \delta)}{\Gamma(p \delta-\beta)}\right)^{\frac{1}{p q-1}}$,

where $\delta$ and $\xi$ are defined in (35).

Let us notice at this stage that the analysis in [3] gives no information on $T_{\max }^{\alpha, \beta}$ or $\tau_{\text {max }}^{\alpha, \beta}$.

Now, let $e_{\alpha}(t):=E_{\alpha, 1}\left(-t^{\alpha}\right)$ where $E_{\alpha, \beta}(t)$ is the Mittag-Leffler function of two parameters $\alpha$ and $\beta$.

Lemma 3.3. The solution $(u, v)$ to the system (FDS) satisfies

$$
\begin{aligned}
& u(t)=u_{0}+\int_{0}^{t} e_{1-\alpha}(t-\tau)|v(\tau)|^{q} d \tau, \\
& v(t)=v_{0}+\int_{0}^{t} e_{1-\beta}(t-\tau)|u(\tau)|^{p} d \tau .
\end{aligned}
$$


Proof. Taking the Laplace transform of both sides of the first equation of the system (FDS) and using (9) and (13), we obtain

$$
\mathcal{L}\{u(t): s\}=\frac{u_{0}}{s}+\frac{\mathcal{L}\left\{|v(t)|^{q}: s\right\}}{\left(s+s^{\alpha}\right)},
$$

which, via the inverse Laplace transform, leads to:

$$
u(t)=u_{0}+\mathcal{L}^{-1}\left(\frac{\mathcal{L}\left\{|v(t)|^{q}: s\right\}}{\left(s+s^{\alpha}\right)}\right)
$$

Notice that

$$
\frac{\mathcal{L}\left\{|v(t)|^{q}: s\right\}}{\left(s+s^{\alpha}\right)}=\frac{s^{-\alpha}}{\left(s^{1-\alpha}+1\right)} \mathcal{L}\left\{|v(t)|^{q}: s\right\}
$$

by means of the inverse Laplace transform of convolution of functions, equation (37) is obtained. Following the same steps, we obtain equation (38).

Lemma 3.4. For the system $(F D S)$, the functions $u^{\prime}(t)$ and $v^{\prime}(t)$ satisfy

$$
\begin{aligned}
& u^{\prime}(t)=|v(t)|^{q}+\int_{0}^{t} e_{1-\alpha}^{\prime}(t-\tau)|v(\tau)|^{q} d \tau, \\
& v^{\prime}(t)=|u(t)|^{p}+\int_{0}^{t} e_{1-\beta}^{\prime}(t-\tau)|u(\tau)|^{p} d \tau .
\end{aligned}
$$

Proof. By virtue of the definition of the Caputo derivative, for $0<\alpha<1$, the equation corresponding to $u(t)$ in system (FDS) takes the form

$$
u^{\prime}(t)+J_{0_{+}}^{1-\alpha} u^{\prime}(t)=|v(t)|^{q}, \quad t>0,
$$

which can be written as

$$
u^{\prime}(t)=\left(1+J_{0_{+}}^{1-\alpha}\right)^{-1}|v(t)|^{q}, \quad t>0 .
$$

Since

$$
\left(1+J_{0_{+}}^{1-\alpha}\right)^{-1}=1+\sum_{k=1}^{+\infty}(-1)^{k} J^{(1-\alpha) k}
$$

we have

$$
\begin{aligned}
& u^{\prime}(t)=|v(t)|^{q}+\left(\sum_{k=1}^{+\infty}(-1)^{k} \frac{t^{(1-\alpha) k-1}}{\Gamma((1-\alpha) k)}\right) \star|v(t)|^{q}, \\
&=|v(t)|^{q}+\int_{0}^{t} e_{1-\alpha}^{\prime}(t-\tau)|v(\tau)|^{q} d \tau, \\
& 10
\end{aligned}
$$


where relations (5) and (6) have been used.

Let us mention that the same result can be obtained by using the Laplace transform technique: taking the Laplace transform of both sides of equation (41), it follows

$$
\mathcal{L}\left\{u^{\prime}(t): s\right\}=\frac{s^{1-\alpha} \mathcal{L}\left\{|v(t)|^{q}: s\right\}}{s^{1-\alpha}+1} .
$$

There are different ways of writing the solution from equation (43); for example we can write

$$
\frac{s^{1-\alpha} \mathcal{L}\left\{|v(t)|^{q}: s\right\}}{s^{1-\alpha}+1}=s\left(\frac{s^{-\alpha} \mathcal{L}\left\{|v(t)|^{q}: s\right\}}{s^{1-\alpha}+1}\right)
$$

and we obtain

$$
u^{\prime}(t)=\frac{d}{d t}\left(e_{1-\alpha} \star|v(t)|^{q}\right) .
$$

Another way of writing equation (43) is

$$
\mathcal{L}\left\{u^{\prime}(t)\right\}=\frac{s^{-\alpha}}{s^{1-\alpha}+1}\left(s \mathcal{L}\left\{|v(t)|^{q}: s\right\}-|v(0)|^{q}\right)+\frac{s^{1-\alpha}}{s^{-\alpha}+1}|v(0)|^{q} ;
$$

taking the inverse Laplace transform, we get

$$
u^{\prime}(t)=\int_{0}^{t} \frac{d}{d t}\left(|v(t-\tau)|^{q}\right) e_{\alpha}(\tau) d \tau+|v(0)|^{q} e_{\alpha}(t) .
$$

Noting that $e_{1-\alpha}(0)=E_{1-\alpha, 1}(0)=1$, equation (43) can be written as

$$
\mathcal{L}\left\{u^{\prime}(t): s\right\}=\left(\frac{s^{1-\alpha}}{s^{1-\alpha}+1}-1\right)|v(s)|^{q}+|v(s)|^{q},
$$

hence

$$
u^{\prime}(t)=\int_{0}^{t}|v(\tau)|^{q} e_{1-\alpha}^{\prime}(t-\tau) d \tau+|v(t)|^{q} .
$$

The equation (40) can be obtained similarly.

\subsection{Analysis of the results}

It is a well known fact [4] that the Mittag-Leffler function $E_{1-\alpha, 1}\left(-t^{1-\alpha}\right)=$ : $e_{1-\alpha}(t)$ is completely monotone for $t>0,0<\alpha<1$; so, in particular $e_{1-\alpha}^{\prime}(t) \leq 0$ for $t>0$. Then equations (39) and (40) allow us to write 


$$
u^{\prime}(t) \leq|v(t)|^{q}, \quad v^{\prime}(t) \leq|u(t)|^{p}, \quad t>0 .
$$

The differential inequalities (44) lead to the estimates from above of the solution $(u, v)$ for $0<t<T_{\max }<+\infty$

$$
u(t) \leq C_{1}\left(T_{\max }-t\right)^{-\frac{q+1}{p q-1}}, \quad v(t) \leq C_{2}\left(T_{\max }-t\right)^{-\frac{p+1}{p q-1}},
$$

where $T_{\max }, C_{1}$ and $C_{2}$ are given in (20). To obtain estimates from below for $u$ and $v$, we notice that system (FDS) can be written as:

$$
D_{0_{+}}^{\alpha}\left(u-u_{0}\right)(t)=|v(t)|^{q}-u^{\prime}(t), \quad D_{0_{+}}^{\beta}\left(v-v_{0}\right)(t)=|u(t)|^{p}-v^{\prime}(t), \quad t>0 .
$$

Using inequalities (44) we obtain

$$
D_{0_{+}}^{\alpha}\left(u-u_{0}\right)(t) \geq 0, \quad D_{0_{+}}^{\beta}\left(v-v_{0}\right)(t) \geq 0 .
$$

Lemma 3.5. For any $u_{0}>0, v_{0}>0$, it holds:

$$
u^{\prime}>0, \quad v^{\prime}>0 .
$$

Proof. Let $u_{-}^{\prime}=\max \left(0,-u^{\prime}\right), u_{+}^{\prime}=\max \left(0, u^{\prime}\right), v_{-}^{\prime}=\max \left(0,-v^{\prime}\right), v_{+}^{\prime}=\max \left(0, v^{\prime}\right)$, so, $u^{\prime}=u_{+}^{\prime}-u_{-}^{\prime}$ and $v^{\prime}=v_{+}^{\prime}-v_{-}^{\prime}$. We are intended to show that $u_{-}^{\prime}=0$ and $v_{-}^{\prime}=0$. From system (FDS) and using $D D_{0_{+}}^{\alpha}=D_{0_{+}}^{\alpha} D$ (where $D$ is the first order integer derivative) we have

$$
\begin{aligned}
u^{\prime \prime}+D_{0_{+}}^{\alpha} u^{\prime} & =q v^{q-1} v^{\prime}, \\
v^{\prime \prime}+D_{0_{+}}^{\beta} v^{\prime} & =p u^{p-1} u^{\prime} .
\end{aligned}
$$

Multiplying both sides of the equation (46) with $u_{-}^{\prime}$ and using the definitions of $u_{+}^{\prime}$ and $u_{-}^{\prime}$, we get the equation

$$
\left(u_{-}^{\prime \prime}\right)\left(u_{-}^{\prime}\right)+\left(u_{-}^{\prime}\right) D_{0_{+}}^{\alpha}\left(u_{-}^{\prime}\right)=-q v^{q-1} v^{\prime}\left(u_{-}^{\prime}\right) .
$$

Taking the integral over $[0, T]$ and using the inequality $\int_{0}^{T} w(t) D_{0_{+}}^{\alpha} w \geq 0, w(0)=$ $0,0<\alpha<1$ [5], the following inequality is obtained

$$
\int_{0}^{T}\left(u_{-}^{\prime \prime}\right)\left(u_{-}^{\prime}\right) \leq-q \int_{0}^{T} v^{q-1} v^{\prime}\left(u_{-}^{\prime}\right),
$$




$$
\begin{aligned}
& =-q \int_{0}^{T} v^{q-1} v_{+}^{\prime}\left(u_{-}^{\prime}\right)+q \int_{0}^{T} v^{q-1} v_{-}^{\prime}\left(u_{-}^{\prime}\right), \\
& \leq q \int_{0}^{T} v^{q-1} v_{-}^{\prime}\left(u_{-}^{\prime}\right),
\end{aligned}
$$

Using Hölder's inequality, we obtain

$$
\begin{aligned}
\frac{d}{d t}\left(\frac{1}{2} \int_{0}^{T}\left(u_{-}^{\prime}\right)^{2}\right) & \leq q C(T) \epsilon \int_{0}^{T}\left(v_{-}^{\prime}\right)^{2}+q C(T) C^{*}(\epsilon) \int_{0}^{T}\left(u_{-}^{\prime}\right)^{2} \\
& \leq C_{1}\left(\int_{0}^{T}\left(v_{-}^{\prime}\right)^{2}+\int_{0}^{T}\left(u_{-}^{\prime}\right)^{2}\right)
\end{aligned}
$$

where $C_{1}=\max \left\{q C(T) \epsilon, q C(T) C^{*}(\epsilon)\right\}$, since $v$ is locally bounded. Likewise, we obtain

$$
\frac{d}{d t}\left(\frac{1}{2} \int_{0}^{T}\left(v_{-}^{\prime}\right)^{2}\right) \leq C_{2}\left(\int_{0}^{T}\left(v_{-}^{\prime}\right)^{2}+\int_{0}^{T}\left(u_{-}^{\prime}\right)^{2}\right) .
$$

Adding (49) and (50), we obtain

$$
\frac{d}{d t}\left(\int_{0}^{T}\left(u_{-}^{\prime}\right)^{2}+\left(v_{-}^{\prime}\right)^{2}\right) \leq C\left(\int_{0}^{T}\left(v_{-}^{\prime}\right)^{2}+\int_{0}^{T}\left(u_{-}^{\prime}\right)^{2}\right),
$$

where $C=\max \left(2 C_{1}, 2 C_{2}\right)$. Using Gronwall's inequality and the fact that $u_{-}^{\prime}(0)=$ $v_{-}^{\prime}(0)=0$, we conclude that $u_{-}^{\prime}=0$ and $v_{-}^{\prime}=0$; hence the result.

At this stage, the following configuration is obtained

$$
\begin{array}{cc}
D_{0_{+}}^{\alpha}\left(u-u_{0}\right)(t) \leq|v(t)|^{q}, & D_{0_{+}}^{\beta}\left(v-v_{0}\right)(t) \leq|u(t)|^{p}, \\
u^{\prime}(t) \leq|v(t)|^{q}, & v^{\prime}(t) \leq|u(t)|^{p} .
\end{array}
$$

Furthermore, the system (FDS) along with $u^{\prime}>0, v^{\prime}>0$ and $D_{0_{+}}^{\alpha}\left(u-u_{0}\right)(t) \geq$ $0, D_{0_{+}}^{\beta}\left(v-v_{0}\right)(t) \geq 0$ allow us to write

$$
\text { either } \quad u^{\prime}(t) \geq \frac{1}{2}|v(t)|^{q} \quad \text { or } \quad D_{0_{+}}^{\alpha}\left(u-u_{0}\right)(t) \geq \frac{1}{2}|v(t)|^{q},
$$


and

$$
\text { either } \quad v^{\prime}(t) \geq \frac{1}{2}|u(t)|^{p} \quad \text { or } \quad D_{0_{+}}^{\beta}\left(u-u_{0}\right)(t) \geq \frac{1}{2}|u(t)|^{p} .
$$

The analysis of the above inequalities gives us the precise profile of the blowing up solutions of system (FDS).

Remark 3.6. The lack of estimates on the blow up time for (PFDS) prevent us to have bilateral estimates on the blow up time for the solutions of (FDS).

\section{Numerical implementation}

For the numerical treatment of the system (FDS), we will approximate the solution $(u, v)$ through equations (37) and (38). The approximation of solutions to the system (PFDS) is obtained via its equivalent form given by the system of Volterra equations in (32).

The numerical approximations of the solutions to both systems (FDS) and (PFDS) require the approximations of the convolution integrals; this has been studied to a reasonable extent in the literature. One of the techniques is the convolution quadrature method in which the quadrature weights are determined by the Laplace transform of the convolution kernel and a linear multistep method (see [6] and references therein).

In this work, the backward Euler convolution quadratures are used for approximating the convolution integral

$$
\int_{0}^{t} K(t-\tau) g(\tau) d \tau .
$$

Let $\varrho>0$ be the time step of the discretization, then

$$
\begin{aligned}
\int_{0}^{t} K(t-\tau) g(\tau) d \tau & =\int_{0}^{t}\left(\frac{1}{2 \pi i} \int_{\gamma} e^{s(t-\tau)} \mathcal{L}\{K(\tau): s\} d s\right) g(\tau) d \tau \\
& =\frac{1}{2 \pi i} \int_{\gamma} y(s, t) \mathcal{L}\{K(\tau): s\} d s,
\end{aligned}
$$

where $\gamma$ is a suitable path connecting $-i \infty$ to $+i \infty$ and

$$
y(s, t)=\int_{0}^{t} e^{s(t-\tau)} g(\tau) d \tau
$$


is the solution of the initial value problem

$$
y^{\prime}=s y+g, \quad y(0)=0, \quad 0 \leq t \leq T .
$$

The backward Euler convolution quadrature is obtained as

$$
\int_{0}^{t_{n}} K\left(t_{n}-s\right) u(s) \sim \frac{1}{2 \pi i} \int_{\gamma} \mathcal{L}\{K(\tau): s\} y_{n}(s) d s,
$$

where $t_{n}=n \varrho$, and $y_{n}(s)$ stands for the approximation of $y\left(s, t_{n}\right)$ reached by the backward Euler method applied to (54). Therefore, if $\delta(\sigma)$ is the generating polynomial for the backward Euler method that is $\delta(\sigma)=1-\sigma$, then

$$
\int_{0}^{t_{n}} K\left(t_{n}-\tau\right) g(\tau) d \tau \sim \sum_{j=0}^{n} q_{n-j}^{\alpha} g\left(t_{j}\right)
$$

the convolution quadrature weights $q_{j}^{\alpha}$ are given by the coefficients of

$$
\sum_{j=0}^{+\infty} q_{j}^{\alpha} \sigma^{j}=\mathcal{L}\left\{K(t): \frac{\delta(\sigma)}{\varrho}\right\} .
$$

For more details on the convolution quadrature methods one is referred to [6] and references therein.

Let for $n \geq 0$ the approximation of $u\left(t_{n}\right)$ be $u_{n}$, then the discritization of the system (FDS) via equations (37), (38) by the backward Euler convolution quadrature method gives

$$
\begin{aligned}
& u_{n}=\left(1-q_{0}^{\alpha} q_{0}^{\beta}\right)^{-1}\left[u_{0}+q_{0}^{\alpha} v_{0}+\sum_{j=0}^{n-1} q_{n-j}^{(\alpha)} v_{j}+q_{0}^{\alpha}\left(\sum_{j=0}^{n-1} q_{n-j}^{\beta} u_{j}\right)\right], \\
& v_{n}=\left(1-q_{0}^{\alpha} q_{0}^{\beta}\right)^{-1}\left[v_{0}+q_{0}^{\beta} u_{0}+\sum_{j=0}^{n-1} q_{n-j}^{(\alpha)} v_{j}+q_{0}^{\beta}\left(\sum_{j=0}^{n-1} q_{n-j}^{\alpha} u_{j}\right)\right],
\end{aligned}
$$

for $n=1,2,3, \ldots$, where the quadrature coefficients $q_{j}^{\alpha}, q_{j}^{\beta}$ are determined from equation (56) with $K(t)=e_{1-\alpha}$ and $K(t)=e_{1-\beta}$, respectively. Similarly, a numerical scheme for the system (PFDS) can be obtained.

\section{Example 1:}

For the figure 1 we set $p=1.5, q=2, \alpha=0.75, \beta=0.5$; the initial conditions are 
$u_{0}=3, v_{0}=2$. In fig(1) (a) the solution curves $u(t)$ corresponding to the systems (FDS), (PFDS) and (ODS) are plotted. The dotted curve is the solution of the system (ODS) with $\lambda=1$. As expected, the middle solid curve corresponds to the solution of the system (FDS). Finally, the dash followed by a dot curve is the solution of the system (PFDS) with $\mu=1 / 2$.

Likewise, in fig(1) (b) the solution curves $v(t)$ for the systems are plotted. It could be seen that the solution curves of the system (FDS) are between the solution curves of (ODS) and (PFDS).

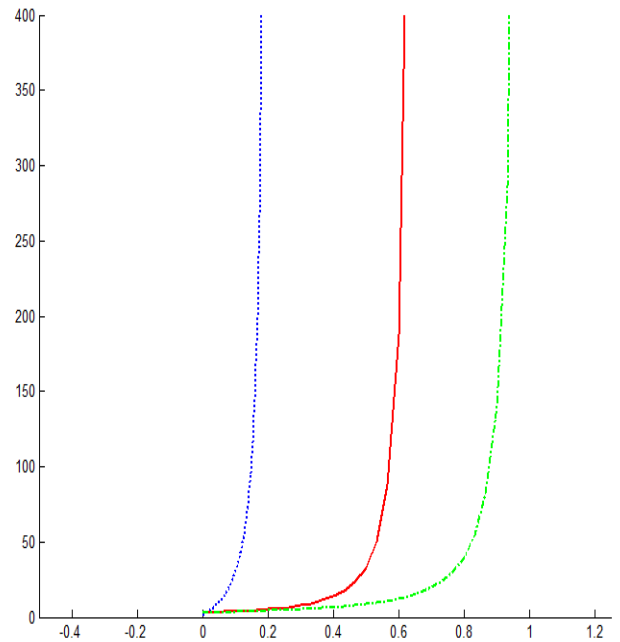

(a)

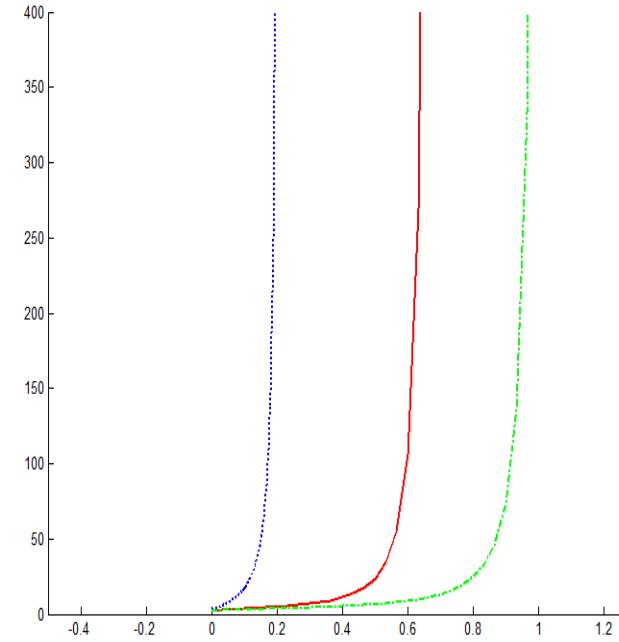

(b)

Figure 1: Solution curves for $p=1.5, q=2, \alpha=0.75, \beta=0.5, u_{0}=3, v_{0}=2$ (a) Solution curves $u(t)$ for the systems (FDS), (PFDS) and (ODS) (b) Solution curves $v(t)$ for the systems (FDS), (PFDS) and (ODS).

\section{Example 2:}

In the second example, we take the parameters $p=1.1, q=1.4, \alpha=0.5=\beta$; the initial conditions $u_{0}=3=v_{0}$. In fig(2) (a) the solution curves $u(t)$ corresponding to the systems (FDS), (PFDS) and (ODS) are plotted. The dotted curve (which serve as lower bound for the solution curve of (FDS)) is the solution of the system (ODS) with $\lambda=1 / 2$. The solid curve is the solution of the system (FDS) and the dash followed by dot curve is the solution of the system (PFDS) with $\mu=1$.

In fig(2) (b) the corresponding solution curves $v(t)$ for the systems are plotted. The profiles of the solution curves for the system (FDS) match the analysis of 
section 3 .

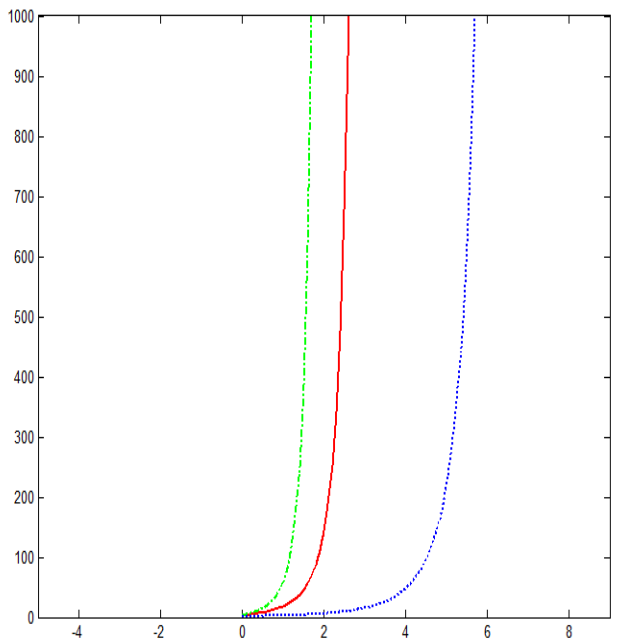

(a)

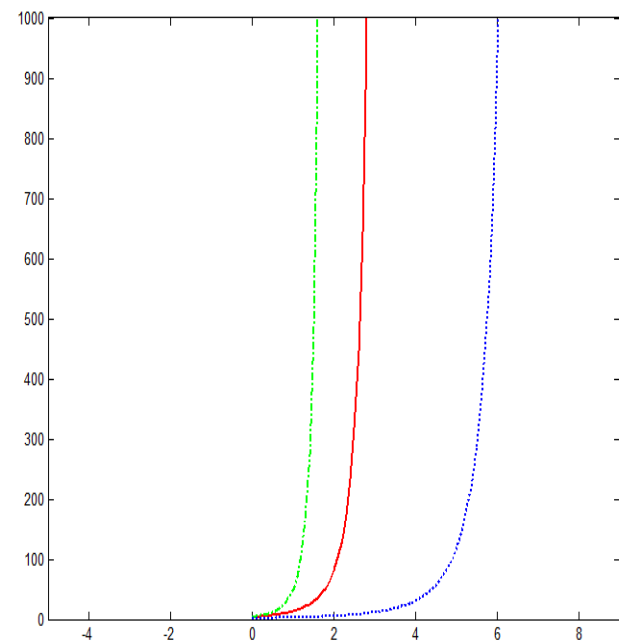

(b)

Figure 2: Solution curves for $p=1.1, q=1.4, \alpha=0.5, \beta=0.5, u_{0}=3, v_{0}=3$ (a) Solution curves $u(t)$ for the systems (FDS), (PFDS) and (ODS) (b) Solution curves $v(t)$ for the systems (FDS), (PFDS) and (ODS).

\section{Example 3:}

For figure 3, we set $p=1.1, q=1.8, \alpha=0.25, \beta=0.4$; the initial conditions are $u_{0}=5, v_{0}=1$. The profile of the solution $(u, v)$, for the system (FDS) is given by the system (ODS) with $\lambda=1$ and the system (PFDS) with $\mu=1 / 2$.

As expected, our simulations show a dependance between the parameters $\left(\alpha, \beta, u_{0}, v_{0}, p, q\right)$ and the calculated blow up.

\section{References}

[1] K. M. Furati, M. Kirane, Necessary conditions for the existence of global solutions to systems of fractional differential equations, Fractional Calculus \& Applied Analysis 11 (2008) 281-298.

[2] A. A. Kilbas, H. M. Srivastava, J. J. Trujillo, Theory and Applications of Fractional Differential Equations, Elsevier, 2006.

[3] W. E. Olmstead, C. A. Roberts and K. Deng, Coupled Volterra equations with blow-up solutions, Journal of Integral Equations \& Applications 7 (1995) 499-516.

[4] H. Pollard, The completely monotonic character of the Mittag-Leffler function $E_{a}(-x)$, Bull. Amer. Math. Soc. 54 (1948) 1115-1116. 


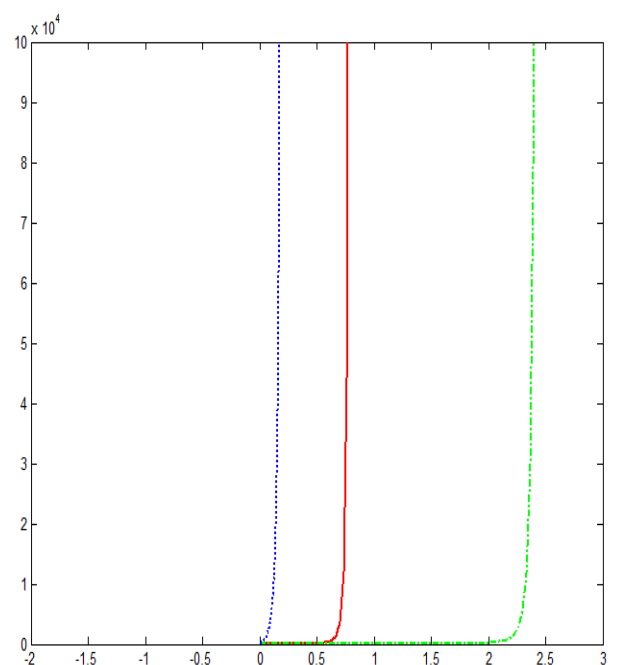

(a)

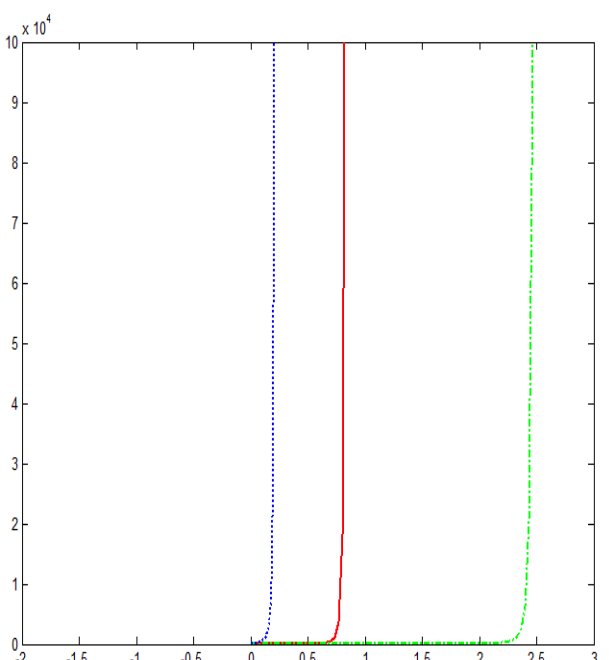

(b)

Figure 3: Solution curves for $p=1.1, q=1.8, \alpha=0.25, \beta=0.4, u_{0}=5, v_{0}=1$ (a) Solution curves $u(t)$ for the systems (FDS), (PFDS) and (ODS) (b) Solution curves $v(t)$ for the systems (FDS), (PFDS) and (ODS).

[5] B. Stankovich, T. M. Atanackovic, On an inequality arising in fractional oscillator theory, Fractional Calculus \& Applied Analysis 7 (2004) 11-20.

[6] C. Lubich: Convolution quadrature revisited. BIT 44, 503-514 (2004) 